\title{
Unreliable trains and induced rescheduling: implications for cost-benefit analysis
}

\author{
Yin-Yen Tseng • Piet Rietveld • Erik T. Verhoef
}

Published online: 24 May 2011

(C) The Author(s) 2011. This article is published with open access at Springerlink.com

\begin{abstract}
This paper is concerned with the assessment of generalized user cost reductions in the cost-benefit analysis (CBA) of transport policies that aim at reducing unreliability. In particular, we investigate the implications of railway passengers' anticipating departure behavior when train services were unreliable. A simple model is established to describe and predict such anticipating behavior. Our numerical example shows how travelers' anticipating departures and scheduling costs depend on the level of unreliability. The possible bias incurred by ignoring the reliability and schedule delay costs reductions in the traditional CBA can be quite substantial. Given our assumptions and parameterization, the underestimation of ignoring these costs could range from 33 to $75 \%$ of total generalized user cost reductions.
\end{abstract}

Keywords Train reliability $\cdot$ Schedule delay - Departure time choice $\cdot$ Anticipating behavior · Public transport $\cdot$ Cost-benefit analysis

\section{Introduction}

Departure time choice is an important element in travelers' decision making, and it becomes more complicated when the travel time is unreliable. As the degree of travel time unreliability increases, travelers can be expected to shift their departure times to earlier moments to compensate for the increased probability of being late (Gaver 1968; Knight 1974; Polak 1987). How early a traveler would shift his departure schedule depends on how he makes tradeoffs among early arrivals, late arrivals, and travel time (in the case

\footnotetext{
Y.-Y. Tseng $(\varangle) \cdot$ P. Rietveld $\cdot$ E. T. Verhoef

Department of Spatial Economics, VU University Amsterdam, De Boelelaan 1105,

1081 HV Amsterdam, The Netherlands

e-mail: ytseng@feweb.vu.nl

P. Rietveld

e-mail: prietveld@feweb.vu.nl

E. T. Verhoef

e-mail: everhoef@feweb.vu.nl
} 
when travel time varies by time of day), for uncertain travel times. An optimal departure time can be expected to be chosen, such that the traveler's resulting expected utility is maximized (Noland and Small 1995).

The choice of departure time is of great interest in public transport. Unlike road transport, where the departure time can be chosen freely, the public transport travelers can only choose between fixed scheduled services, as defined by the timetable. Thus, it is common that train travelers may choose to take an earlier than necessary connection to reduce the probability of arriving too late. It is similar to the situation in road transport where people may reserve a 'safety margin' for unexpected delays in traffic, but in public transport the departure time is usually discrete, and restricted by the timetable. Though travelers may have other reactions to cope with unreliable train services, e.g., shifting to other modes, in the present study we focus on the choice of shifting of departure time for train users. We refer to the behavior of taking an earlier than strictly necessary train connection as "anticipating departure". We will develop a simple model to investigate how the traveler makes the decision between alternative scheduled services when unreliability is involved.

This paper builds on a number of earlier contributions. In modeling the effect of travel time reliability, scheduling preferences are generally used to analyze travelers' departure time choice behavior, following, e.g., Vickrey (1969), Small (1982), and Noland and Small (1995). Bates et al. (2001) concluded that for car travel, the expected scheduling cost can be well approximated by a function that is linear in the standard deviation of the travel time distribution. Later, Fosgerau and Karlström (2010) provided a unified theoretical framework, bridging the gap between the scheduling model and the mean-variance model. They found that the optimized expected cost is linear in the mean and standard deviation for a standardized travel time distribution. However, they found no general explicit solution for general travel time distributions for scheduled services, but concluded that travelers' expected cost function is not linear in the mean and standard deviation of travel time distribution.

Since the reliability of travel times is becoming increasingly important for policy evaluation, it is important that its valuation can be incorporated into cost-benefit analyses (CBAs) of infrastructure projects. Nevertheless, given that there is no direct link between the scheduling model and the mean-variance model for scheduled services, it is not straightforward how the scheduling parameters can be used in a CBA framework.

Our paper focuses on scheduled services, and derives the expected schedule delay cost under some specific circumstances. We allow for departure time (connection) choice for unreliable services, and find numerical and analytical solutions for specific cases. In particular, we take travelers' "anticipating departures" (departing earlier than strictly necessary in response to travel time unreliability) into account explicitly when deriving the expected scheduling costs. It is the discreteness of departure time choice that makes the analysis different from that for road transport. The outline of the remaining paper is as follows. "The model" section presents the theoretical model for departure time choice with scheduled services in public transport. "Anticipating departure behavior and expected scheduling costs" section discusses the anticipating behavior and derives the expected scheduling costs for the cases of reliable and unreliable services. "Numerical illustrations" section provides numerical illustrations based on the analytical framework developed in previous sections. In "Application in cost-benefit analysis" section we apply the results in the calculation of the reductions in generalized user costs, by incorporating the effects of reliability and schedule delay in a CBA. "Concluding remarks" section then concludes. 


\section{The model}

Suppose a train runs regularly with a headway $H$, and one service is scheduled to arrive at $t=T$. $T$ is chosen such that a traveler's preferred arrival time (PAT) is in the range [T, $T+H]$, so he has to decide whether to catch the connection arriving at $t=T$, a later one arriving at $t=T+H$, or perhaps even another one. We assume that this traveler will choose the connection that gives him the maximum expected utility (or minimum expected disutility). In principle, if the train service is completely reliable, this traveler's choice would either be the service that arrives at $T$ (denoted as train connection B) or the one at $T+H$ (denoted as train connection $\mathrm{C}$ ), depending on his PAT and the values of schedule delay early (SDE) and late. Nevertheless, if the train service is very unreliable, (particularly when the possible delay time approaches the headway), it may be possible that this traveler chooses to take an earlier than necessary connection, with a timetable arrival time at $t=T-H$ (denoted as connection A). Without loss of generality, we can set the clock time $T=0$. Figure 1 then illustrates travelers' PATs and the arrival times of train connections A-C.

We want to study travelers' decisions under these circumstances. The analysis set up for this paper makes the following assumptions:

1. The headway of the train service is $H$, and time $T=0$ is chosen such that travelers' PATs are in the range $[0, H]$;

2. The train scheduled to arrive at $t=-H$ (timetable arrival time, not the actual arrival time) is called connection ' $\mathrm{A}$ '; the train to arrive at $t=0$ is called connection ' $\mathrm{B}$ '; and the train to arrive at $t=H$ is called connection ' $\mathrm{C}$ ';

3. Each train has a probability of being late. More precisely, there is a distribution of $\mathrm{n}$ possible delays $D T_{i}$ (Delay Time), each occurring with a probability $p_{i}$. We indicate a zero delay as $D T_{0}=0$, and observe that $p_{0}=1-\sum_{i=1}^{n} p_{i}$. We assume that $0 \leq D T_{i} \leq H^{1}$

4. Since the trip duration of the train service, unlike in road transport, is usually timeinvariant, the travel time can be normalized to zero without loss of generality. For a train without delay, the departure time and arrival time then coincide.

To derive the relationship between $H, P A T$, and schedule delay parameters, we adopt the functional form proposed by Noland and Small (1995), which has been used extensively in the literature of the departure time choice modeling (see, e.g., Bates et al. 2001; Ettema and Timmermans 2006). It expresses the scheduling disutility as:

$$
D U_{s}=\beta \cdot E[S D E]+\gamma \cdot E[S D L]+\theta \cdot P_{L},
$$

where $D U_{s}$ denotes the disutility associated with scheduling; $E[S D E]$ is the expected SDE; $E[S D L]$ is the expected schedule delay late; and $P_{L}$ is the expected probability of late arrival. The $E[S D E], E[S D L]$, and $P_{L}$ for train connections A-C can be computed as follows:

\footnotetext{
1 The assumption that $D T_{i} \leq H$ facilitates the analysis of travelers' anticipating departures. Since travelers may take the connection that arrives at $t=-2 \mathrm{H}$ or earlier when the delay time DT is larger than $2 \mathrm{H}$ or more, abandoning this assumption would require consideration of a fourth possible service. Therefore, to simplify the analysis for predicting the anticipating departures, we consider the case for which the longest delay time is $\mathrm{H}$, so that a traveler will only have to choose between connections $\mathrm{A}-\mathrm{C}$.
} 


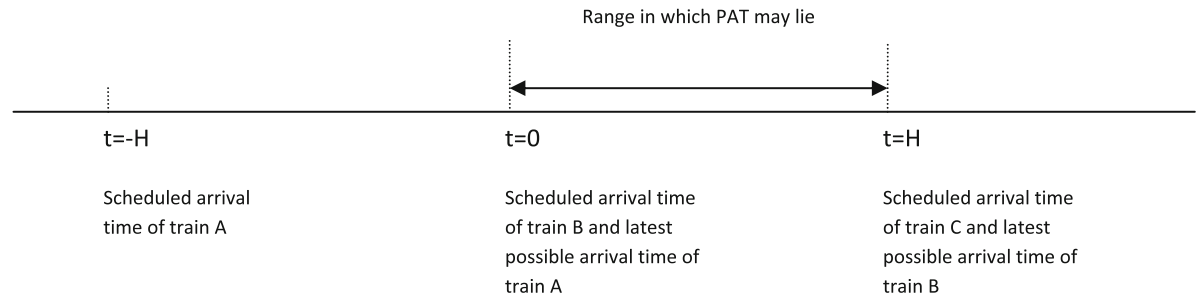

Fig. 1 Travelers' PATs and arrival times of different connections

Train connection A

$$
\begin{aligned}
E[S D E] & =\sum_{i=0}^{n} p_{i} \cdot\left[P A T+H-D T_{i}\right] \\
E[S D L] & =0 \\
P_{L} & =0 .
\end{aligned}
$$

Train connection $B$

If $P A T \geq D T_{n}$, then

$$
\begin{aligned}
E[S D E] & =\sum_{i=0}^{n} p_{i} \cdot\left[P A T-D T_{i}\right] \\
E[S D L] & =0 \\
P_{L} & =0 .
\end{aligned}
$$

Otherwise, define $j$ such that $D T_{j-1} \leq P A T<D T_{j}$. Then:

$$
\begin{aligned}
E[S D E] & =\sum_{i=0}^{j-1} p_{i} \cdot\left[P A T-D T_{i}\right] \\
E[S D L] & =\sum_{i=j}^{n} p_{i} \cdot\left[D T_{i}-P A T\right] \\
P_{L} & =\sum_{i=j}^{n} p_{i} .
\end{aligned}
$$

Train connection $C$

$$
\begin{aligned}
E[S D E] & =0 \\
E[S D L] & =\sum_{i=0}^{n} p_{i} \cdot\left[\left(H+D T_{i}\right)-P A T\right] \\
P_{L} & =1 .
\end{aligned}
$$

The decision rule is that a traveler will choose train connection $k=[\mathrm{A}, \mathrm{B}, \mathrm{C}]$ if the schedule disutility of train $k, D U_{s}(k)$, is the smallest of these three available train connections.

In the following section, we discuss how we apply this model to describe the anticipating departure behavior and to calculate the expected scheduling cost, under different levels of reliability of train services. 


\section{Anticipating departure behavior and expected scheduling costs}

Unreliable train service and travelers' anticipating departure

In public transport, it is common that people may choose an earlier than necessary connection, according to the timetable, in order to reduce the probability of late arrival. This kind of behavior is referred to as 'anticipating departure' in the present study. Here, we will explore the influence of unreliability on train users' anticipating departures, in the context of the conceptual framework presented in the previous section.

Let us first consider an individual's departure time choice in the case of a completely reliable train service. In that case, connection A will never be taken by a traveler, since it is always dominated by connection B (the SDE of connection A is always larger than it is for connection B). Therefore, a traveler will choose either connection B or C, depending on his PAT and schedule delay parameters. For the sake of simplicity, we assume that $\theta=0$ here. The switching level of PAT, denoted PAT*, for a choice between two reliable train services can then be computed as follows:

$$
\begin{aligned}
& D U_{s}(\text { Connection } \mathrm{B})=D U_{s}(\text { Connection } \mathrm{C}) \\
& \Rightarrow \beta \cdot P A T^{*}=\gamma \cdot\left(H-P A T^{*}\right) \\
& \Rightarrow P A T^{*}=\frac{\gamma \cdot H}{\beta+\gamma}
\end{aligned}
$$

It implies that a traveler will choose to take connection B if his PAT falls in the interval $\left[0, \frac{\gamma \cdot H}{\beta+\gamma}\right]$, and will switch to connection $\mathrm{C}$ if his PAT is in the interval $\left[\frac{\gamma \cdot H}{\beta+\gamma}, H\right]$ under the reliable train service circumstance. This result and Eq. 6 were also obtained by de Palma and Lindsey (2001) and Bates et al. (2001).

Based on the analysis above, we can also compute a traveler's connection choice behavior under travel time unreliability (given the amount of delay times and their associated probabilities). By comparing individuals' connection choices between unreliable services with the choices between reliable services, we are able to determine whether a traveler chooses an anticipating departure or not. In other words, if a traveler takes connection B with a completely reliable train service, and chooses connection A, for another unreliable set of services, then he has made an "anticipating departure". Similarly, the anticipating departure also exists when connection $\mathrm{C}$ is chosen under reliable services, and $\mathrm{A}$ or $\mathrm{B}$ is chosen under unreliable services.

Expected scheduling costs for regular and scheduled transport services

\section{Expected scheduling costs associates with reliable services}

Note that the scheduling disutility is generally not zero even for a completely reliable service. That is, whenever the train company does not provide the service that fits every traveler's PAT: for example, because PATs vary over travelers, there is always some disutility associated with scheduling inconvenience. Since we can compute the scheduling disutilities for the reliable train service under different levels of headway $H$, the "value of headway' as it will be called can also be derived in our framework.

In "Unreliable train service and travelers' anticipating departure" section, we obtained that the switching level of PAT* for a choice between two reliable train connections is $\frac{\gamma \cdot H}{\beta+\gamma}$. 
A traveler chooses to take connection B if his PAT is earlier than PAT*, and connection $\mathrm{C}$ if his PAT is later than PAT*. In such a case, $E[S D E]=P A T$ and $E[S D L]=H-P A T$, when $0 \leq P A T \leq \frac{\gamma \cdot H}{\beta+\gamma} ; E[S D E]=0$ and $E[S D E]=H-P A T$, otherwise.

The expected scheduling costs for this reliable service can then be derived analytically for uniformly distributed PATs lying between 0 and $H .{ }^{2}$ To simplify the derivation, we again assume that $\theta=0$. The derivation for the expected (average) scheduling costs, and its marginal value with respect to the headway, are shown in Eqs. 7 and 8, respectively:

$$
\begin{gathered}
E\left[D U_{s}\right]^{R}=\frac{1}{H}\left[\int_{0}^{\frac{\gamma \cdot H}{\beta+\gamma}} \beta \cdot P A T \cdot d P A T+\int_{\frac{\gamma \cdot H}{\beta+\gamma}}^{H} \gamma \cdot(H-P A T) \cdot d P A T\right]=\frac{\beta \cdot \gamma}{2(\beta+\gamma)} H \\
\frac{\partial E\left[D U_{s}\right]^{R}}{\partial H}=\frac{\beta \cdot \gamma}{2(\beta+\gamma)} .
\end{gathered}
$$

Note that Eq. 7 follows immediately as half the maximum schedule delay cost for the traveler at $\mathrm{PAT}^{*}$, which is $\beta \cdot P A T^{*}=\beta \cdot \gamma /(\beta+\gamma) \cdot H$. The "half" stems from the linearity of schedule delay costs.

Equation 7 is also given in de Palma and Lindsey (2001), Wardman (2004), and Fosgerau (2009), where it is defined as the average headway disutility due to schedule inconvenience, and Eq. 8 can be regarded as the value of headway implied by schedule delay costs. It is clear that this headway value depends on the VSDE and VSDL, and it is intuitively plausible that higher values of schedule delay parameters would result in a higher value of headway (Eq. 8 is always positive and so are its derivatives w.r.t. $\beta$ and $\gamma$ ).

\section{Expected scheduling costs generated from unreliable services}

In the previous subsection, we showed how to calculate the expected schedule delay costs for reliable services. We can now calculate the expected schedule delay costs for unreliable train services in a similar way. By taking the difference of expected schedule delay costs between reliable and unreliable services, we are able to obtain the change in generalized user cost associated with an improvement of service reliability. In determining the expected schedule delay costs for unreliable services, one crucial aspect in modeling is the travelers' departure connection choice. More specifically, if travelers are assumed to take the same connection for unreliable services as for reliable ones, the expected schedule delay costs will be different from the situation where travelers have anticipating departure, implying that travelers may change their departure behavior according to the levels of unreliability.

To illustrate the difference of computing the expected schedule delay costs in these two situations, we demonstrate two examples of the schedule delay cost functions for fixed and anticipating departures in Figs. 2 and 3, respectively. In these two examples, headway is assumed to be $30 \mathrm{~min}$, and the unreliable train is assumed to provide the service with a two mass-points travel time distribution with a $70 \%$ probability of arrival according to the schedule and a $30 \%$ probability of a 20 -min delay. For parameters of travelers' generalized

\footnotetext{
${ }^{2}$ For some experienced travelers such as commuters who may be able to plan their activities at the destination around the train arrival time, their PATs may in fact be very close to the reliable train arrival times. In such a case, we may assume different distribution of PATs rather than the uniform distributed PATs lying between 0 and $\mathrm{H}$. Here, we only consider the simplest case for demonstrating the idea.
} 


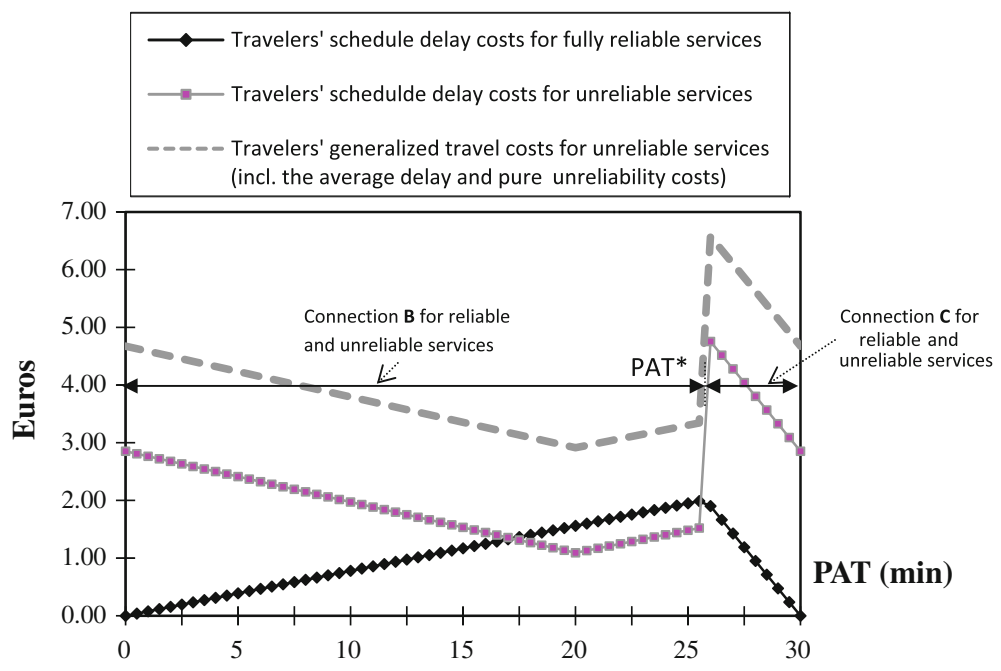

Fig. 2 The schedule delay costs and generalized travel costs for travelers departing as if unreliability does not exist

costs (see Eq. 9), the monetary estimates obtained from Tseng (2008) (see Table 6.5, scheduled commuting trips of full model), with $\alpha=11.71(€ / \mathrm{h}), \sigma=4.26(€ / \mathrm{h}), \beta=4.68$ $(€ / \mathrm{h}), \gamma=28.56(€ / \mathrm{h})$, and $\theta=0$, are used here. The black curves in Figs. 2 and 3 depict the schedule delay costs (Eq. 1) for the fully reliable services. These curves are a function of PAT, depicted along the horizontal axis, and are derived under the assumption that departure time is optimized given PAT (this is, of course, consistent with the definition of a "cost function"). These black curves also represent travelers' generalized travel costs (defined in as Eq. 9), if the travel time and cost are normalized to be zero. Note that PAT* determines the kink in this curve, reflecting that a traveler would choose to take connection $\mathrm{B}$ if his PAT is earlier than PAT*, and take connection $\mathrm{C}$ otherwise.

$$
D U=\alpha \cdot E[T]+\beta \cdot E[S D E]+\gamma \cdot E[S D L]+\sigma \cdot S T D .
$$

The gray curve in Fig. 2 represents the schedule delay cost for unreliable train services under the assumption that travelers' departure connection choice is the same as the one for reliable train services. This is what results when travelers ignore possible delays and just base their departure time on the official timetable. Since PAT* is the point where travelers are indifferent between choosing connections B and C for fully reliable services but not for unreliable services, there is a discrete jump of the cost function in the gray curve at PAT*. This discontinuity reflects that with, unreliable services, PAT* is not the optimal switching point between services B and C. Because there is a probability of delay, travelers with a PAT equal to $\mathrm{PAT}^{*}$ are now still better-off with the earlier connection $\mathrm{B}$, and therefore would face discretely higher expected scheduling costs when choosing $\mathrm{C}$, whereas the gray line only reflects schedule delay costs. Travel delay costs also become relevant when introducing travel time uncertainty, and the same holds for the value of pure unreliability (i.e., $\sigma$ in Eq. 9). The gray dashed line shows the total generalized travel costs (Eq. 9), including the value of average travel delay and of pure unreliability. Note that these additional costs are independent of a traveler's PAT, and therefore shift the earlier gray curves upwards by a constant amount. 


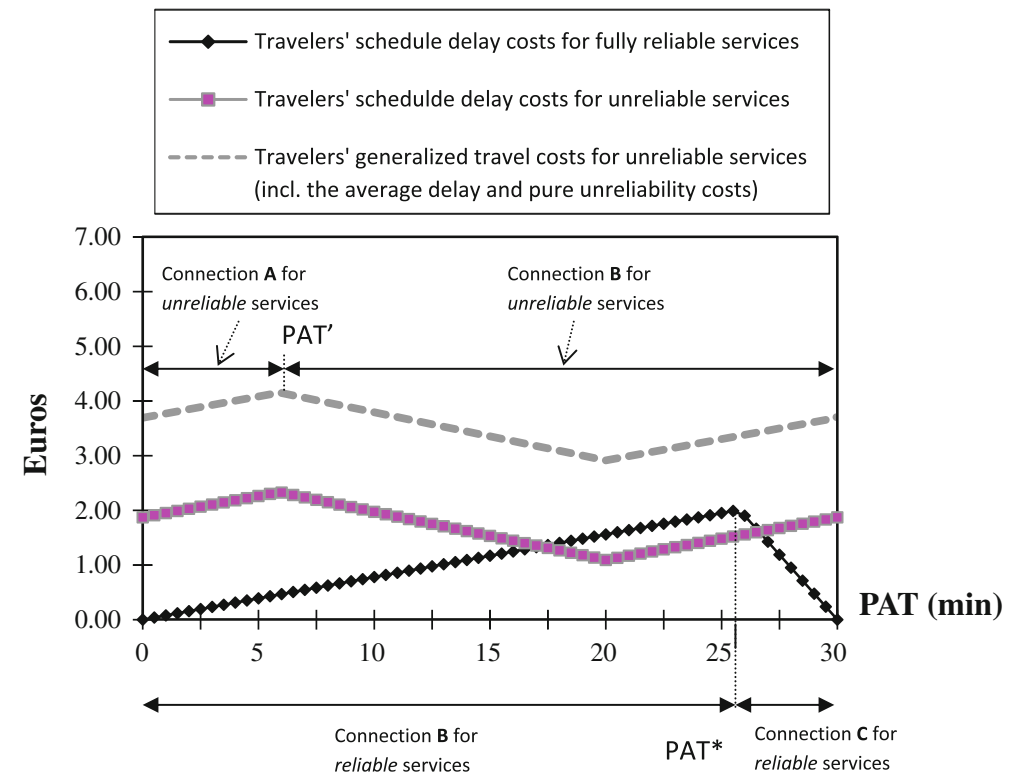

Fig. 3 The schedule delay costs and generalized travel costs for 'anticipating' departure behavior of travelers

Next, the gray curve in Fig. 3 depicts the schedule delay costs for unreliable services when travelers' anticipating choice of departure time is taken into account. In such a case, since travelers will choose the connection that yields the smallest disutility according to the level of unreliability, the resulting connection choice may be different from the case when service is fully reliable. Just as for PAT* in the reliable service, it is also possible to determine the switching level of PAT for the unreliable service. PAT' in Fig. 3 represents the point at which travelers are indifferent between choosing connections A and B in the unreliable services. In other words, a traveler would choose connection A when his PAT is earlier than $\mathrm{PAT}^{\prime}$, otherwise, connection $\mathrm{B}$ is chosen. Note that the gray line is now continuous, indicating that the switch between connections is made at the optimal moment. Again, we also show the generalized travel cost level (gray dashed line) with the value of average travel delay and of pure unreliability included.

Once the schedule delay function has been derived for every relevant PAT, we can easily calculate the expected (average) schedule delay costs for all travelers under both reliable and unreliable situations. By taking the difference of expected schedule delay costs between these two services, the average additional schedule delay costs due to a particular level of unreliability can be obtained. In the case of anticipating departures, this additional expected schedule delay costs can be obtained as Eq.(10) and Eq.(11), depending on whether delay time (DT) is smaller or larger than $\frac{\beta \cdot H}{(\beta+\gamma) \cdot p}$.

When $D T<\frac{\beta \cdot H}{(\beta+\gamma) \cdot p}$

$$
\Delta E\left[D U_{s}\right]=E\left[D U_{s}\right]^{U}-E\left[D U_{s}\right]^{R}=\frac{(\beta+\gamma)}{2 H} \cdot p \cdot(1-p) \cdot D T^{2} .
$$

When $D T \geq \frac{\beta \cdot H}{(\beta+\gamma) \cdot p}$ 
Table 1 Percentage of travelers' anticipating departures for various levels of unreliability

\begin{tabular}{llrrrrr}
\hline Headway $=30 \mathrm{~min}, \beta=$ \\
\hline Delay time (min) & \multicolumn{6}{l}{ Delay probability $€ / \mathrm{h}, \gamma=28.53 € / \mathrm{h}$, and $\theta=0$} \\
\cline { 2 - 7 } & $5 \%$ & $10 \%$ & $20 \%$ & $30 \%$ & $40 \%$ & $50 \%$ \\
\hline 5 & 0.8 & 1.7 & 3.3 & 5.0 & 6.7 & 8.3 \\
10 & 1.7 & 3.3 & 6.7 & 10.0 & 13.3 & 19.3 \\
15 & 2.5 & 5.0 & 10.0 & 17.2 & 28.9 & 35.9 \\
20 & 3.3 & 6.7 & 13.3 & 33.8 & 45.6 & 52.6 \\
25 & 4.2 & 8.3 & 27.0 & 51.0 & 62.3 & 69.3 \\
30 & 4.6 & 9.6 & 43.7 & 67.2 & 78.9 & 85.9 \\
\hline
\end{tabular}

$$
\Delta E\left[D U_{s}\right]=E\left[D U_{s}\right]^{U}-E\left[D U_{s}\right]^{R}=\beta \cdot\left(\frac{1}{2} H+(1-p) \cdot D T-\frac{\beta \cdot H}{2(\beta+\gamma) \cdot p}\right)-\frac{\beta \cdot \gamma \cdot H}{2(\beta+\gamma)} .
$$

where $E\left[D U_{s}\right]^{U}$ and $E\left[D U_{s}\right]^{R}$ represent the expected schedule delay costs for unreliable and reliable services, respectively. The detailed derivation is provided in Appendix 1 where both the fixed and anticipating departures were derived separately.

It is clear that neither Eqs. 10 nor 11 is linear in the standard deviation of travel time, which is $D T \cdot \sqrt{p \cdot(1-p)}$ in the case of two mass-points travel time distribution. This justifies the point made by Fosgerau and Karlström (2010) that travelers' expected cost function is not linear in the mean and standard deviation of travel time distribution for scheduled services.

\section{Numerical illustrations}

In order to analyze anticipating departures numerically, and to derive the value of implied scheduling costs, specific assumptions of travel times, travelers' PAT distribution, and estimates of the cost coefficients $\beta, \gamma, \theta$ are needed. We will use the simplest case of a travel time distribution with two mass-points. A series of two-mass-points travel time distributions (with delay times ranging from 5 to $30 \mathrm{~min}$ and probabilities ranging from 5 to $50 \%$ ) will be examined in this section. For parameters of travelers' generalized costs (see Eq. 9), the coefficients obtained from Tseng (2008), mentioned above, will be used. For the distribution of PATs, we assume that it is uniform. Travelers' PATs at the destination stations are therefore independent of the scheduled timetable.

Table 1 summarizes the resulting percentages of travelers' anticipating departures (compared to complete certainty) under various travel time distributions (that is, given various pairs of delay time and delay probability). As may be expected, the percentage of anticipating departures increases as the delay time and/or the delay probability increase. The effect of delay time and probability on the percentage of anticipating departures is nearly linear when the level of delay time and probability are moderate. However, the anticipating percentage increases much more than proportionally when the level of delay time and probability both become very large. In other words, travelers' anticipating departures become rapidly more common when the services become more strongly unreliable. 
Table 2 Values of implied schedule delay (€/h) and SDRs for various levels of unreliability

\begin{tabular}{|c|c|c|c|c|c|c|}
\hline \multicolumn{7}{|c|}{ Parameters used: headway $=30 \mathrm{~min}, \beta=4.68 € / \mathrm{h}, \gamma=28.53 € / \mathrm{h}$, and $\theta=0$} \\
\hline \multirow[t]{2}{*}{ Delay time (min) } & \multicolumn{6}{|c|}{ Delay probability } \\
\hline & $5 \%$ & $10 \%$ & $20 \%$ & $30 \%$ & $40 \%$ & $50 \%$ \\
\hline \multicolumn{7}{|l|}{ Fixed departure } \\
\hline 5 & $0.64(0.05)$ & $0.93(0.08)$ & $1.39(0.12)$ & $1.82(0.16)$ & $2.27(0.19)$ & $2.78(0.24)$ \\
\hline 10 & $1.27(0.11)$ & $1.85(0.16)$ & $2.77(0.24)$ & $3.63(0.31)$ & $4.53(0.39)$ & $5.55(0.47)$ \\
\hline 15 & $1.91(0.16)$ & $2.77(0.24)$ & $4.16(0.36)$ & $5.44(0.46)$ & $6.79(0.58)$ & $8.31(0.71)$ \\
\hline 20 & $2.54(0.22)$ & $3.69(0.32)$ & $5.54(0.47)$ & $7.25(0.62)$ & $9.05(0.77)$ & $11.08(0.95)$ \\
\hline 25 & $3.18(0.27)$ & $4.62(0.39)$ & $6.92(0.59)$ & $9.07(0.77)$ & $11.31(0.97)$ & $13.85(1.18)$ \\
\hline 30 & $3.74(0.32)$ & $5.43(0.46)$ & $8.14(0.70)$ & $10.66(0.91)$ & $13.30(1.14)$ & $16.28(1.39)$ \\
\hline \multicolumn{7}{|c|}{ Anticipating departure } \\
\hline 5 & $0.60(0.05)$ & $0.83(0.07)$ & $1.11(0.09)$ & $1.27(0.11)$ & $1.36(0.12)$ & $1.38(0.12)$ \\
\hline 10 & $1.21(0.10)$ & $1.66(0.14)$ & $2.21(0.19)$ & $2.54(0.22)$ & $2.71(0.23)$ & $2.70(0.23)$ \\
\hline 15 & $1.81(0.15)$ & $2.49(0.21)$ & $3.32(0.28)$ & $3.79(0.32)$ & $3.71(0.32)$ & $3.36(0.29)$ \\
\hline 20 & $2.41(0.21)$ & $3.32(0.28)$ & $4.43(0.38)$ & $4.63(0.40)$ & $4.22(0.36)$ & $3.69(0.32)$ \\
\hline 25 & $3.13(0.27)$ & $4.15(0.35)$ & $5.40(0.46)$ & $5.13(0.44)$ & $4.52(0.39)$ & $3.89(0.33)$ \\
\hline 30 & $3.59(0.31)$ & $4.97(0.42)$ & $6.06(0.52)$ & $5.47(0.47)$ & $4.72(0.40)$ & $4.02(0.34)$ \\
\hline
\end{tabular}

Note: The SDRs are given in the parentheses

Next, under the same hypothesized travel time distributions, headway, and schedule delay parameters, we can calculate the additional expected scheduling cost $\Delta E\left[D U_{S}\right]$ as discussed in "Expected scheduling costs generated from unreliable services" section. We divide this additional expected scheduling cost by its associated standard deviation of travel time, $\Delta E\left[D U_{S}\right] / S T D$, so that the scheduling cost per unit of standard deviation is obtained. The term $\Delta E\left[D U_{S}\right] / S T D$ will be referred to as 'the value of implied schedule delay' (VOISD) in the following discussion.

Table 2 summaries the resulting values of implied schedule delay for various levels of unreliable services, distinguishing between the imaginary situation where travelers would not adjust their departure time due to unreliable travel times (top half), and the situation where they do exhibit anticipating behavior (bottom half). We calculate the ratio between the value of implied schedule delay and the value of time, and refer to this ratio as the 'schedule delay valuation ratio' (SDR), which is analogous to the definition of the reliability ratio (RR) (e.g., Black and Towriss 1993). The resulting ratios for various levels of unreliability are given in the parentheses of Table 2 .

In Table 2 it is clear that the values of implied schedule delay or the SDRs are not constant over different levels of unreliability. Different from the case of car journeys examined by Noland and Small (1995), who found that the value of implied schedule delay is independent of the standard deviation (under certain assumptions of travel time distributions), our results show that the value of implied schedule delay varies with the level of unreliability. In the case of fixed departures, the value of implied schedule delay increases as the unreliability level, either by delay time or delay probability, increases, and the value rises rapidly when the train service becomes extremely unreliable. As for the case of anticipating departures, the variation pattern is different from the one in the case of fixed departures: the resulting value of implied schedule delay does not increase monotonically 
Table 3 An example of the cost reduction contribution for reliability improvement

\begin{tabular}{|c|c|c|c|c|}
\hline & \multicolumn{2}{|c|}{ Fixed departure } & \multicolumn{2}{|c|}{ Anticipating departure } \\
\hline & $\begin{array}{l}\text { Cost } \\
\text { reduction } \\
\text { (in } € \text { ) }\end{array}$ & $\begin{array}{l}\text { Percentage of } \\
\text { total contribution }\end{array}$ & $\begin{array}{l}\text { Cost } \\
\text { reduction } \\
\text { (in } € \text { ) }\end{array}$ & $\begin{array}{l}\text { Percentage of } \\
\text { total contribution }\end{array}$ \\
\hline $\begin{array}{l}\text { Expected travel time cost reduction } \\
(\alpha=11.71 € / \mathrm{h})\end{array}$ & 1.171 & 39.97 & 1.171 & 46.30 \\
\hline $\begin{array}{l}\text { Expected schedule delay cost reduction } \\
\quad(\beta=4.68 € / \mathrm{h} ; \gamma=28.53 € / \mathrm{h})\end{array}$ & 1.108 & 37.82 & 0.707 & 27.97 \\
\hline $\begin{array}{l}\text { Standard deviation cost reduction } \\
(\sigma=4.26 € / \mathrm{h})\end{array}$ & 0.651 & 22.21 & 0.651 & 25.73 \\
\hline Total generalized cost reduction & 2.930 & 100 & 2.529 & 100 \\
\hline
\end{tabular}

Based on a delay probability of $30 \%$ and a delay time of $20 \mathrm{~min}$

with the level of unreliability. In general, this value increases with the level of unreliability when that level is moderate; nevertheless, the value slightly decreases with the delay probability when the probability is more than $30 \%$ in our numerical example. This may be because, when train services are extremely unreliable, most travelers just adjust their departure time to take an earlier connection; consequently, the expected schedule delay costs and the resulting value of schedule delay would decrease instead. Note that, when comparing the results between fixed and anticipating departures, the values overall are lower in the case of anticipating departures. This makes sense, as a traveler with anticipating departure is assumed to choose his departure connection optimally according to the reliability level, so that his expected scheduling cost is minimized.

\section{Application in cost-benefit analysis}

It is of interest to see what our analysis implies for CBA of policies that reduce unreliability. While quantifying travelers' user cost reductions from travel time savings has been a standard practice in the CBA framework (e.g., Chap. 5 in Gómez-Ibáñez et al. 1999), it is not entirely clear how to incorporate the reduction of travelers' other time-related costs from a reliability improvement. Numerous studies have shown that travelers do place significant values on reliability (standard deviation) and schedule delays. Thus, ignoring the valuation of reliability may sometimes seriously limit the accuracy of a CBA, and therewith its relevance.

The improvement of service reliability can result in the reduction of the standard deviation of travel time, as well as the reduction in (expected) schedule delay costs. In "Anticipating departure behavior and expected scheduling costs and Numerical illustrations" sections, we showed how to derive the expected scheduling costs for the cases of fixed and anticipating departures. In this section we investigate the relative shares of travel time, schedule delays, and pure unreliability, in the total reduction in generalized user cost that follows from a reliability improvement.

An example is given in Table 3, in which there is a $30 \%$ probability of having a 20-min delay for an unreliable train service with 30 min headway. By using the same parameters used in the previous section, we are able to decompose generalized user cost reduction into 
savings associated with the expected travel time, expected schedule delay, and standard deviation (pure reliability) reduction when this unreliable service is improved to become a fully reliable one. Since the expected schedule delay costs are different between different departure pattern assumptions, i.e. fixed and anticipating departures as discussed in previous sections, it is of interest to see the impact of taking into account travelers' behavior change on the reduction of schedule delay cost, relative to the total cost reduction. Table 3 shows the cost reductions for each item and their relative contributions to the overall reduction in user cost.

In this example, the reduction in expected travel time costs is smaller than that in schedule delay cost and reliability cost, in both the fixed and the anticipating departures scenarios. Since the travel time savings is usually one of the largest components in the CBA assessment, these results suggest that the typical CBA procedure, which fails to explicitly take into account these other cost reductions from a reliability improvement, may seriously underestimate the outcome. The example in Table 3 shows that the underestimation is about $60 \%$ and $54 \%$ of total generalized user cost reductions for fixed and anticipating departure travelers, respectively. Note that the reduction of schedule delay cost is smaller in the case of anticipating departures than for fixed departures. This implies that, when travelers' anticipating departure is taken into account, the resulting cost reduction from the schedule delay will not be as large as the situation in which travelers' departure behavior is assumed unchanged. This is intuitive: one can reverse the change and then the results show that an increase in unreliability without allowing optimal anticipation induces higher expected schedule delay costs than if this optimization is allowed.

To see how strongly the reliability level influences the shares of the three components of cost reductions, we calculate the percentages of cost reductions for a complete elimination of unreliability, starting at different initial levels of unreliability. Figure 4 demonstrates the variations of these shares when travelers' departure times are assumed fixed under both reliable and unreliable services. Figure 5 shows the same indicators when anticipating departures are taken into account. The numerical percentages are provided in Tables 4 and 5 in Appendix 2. For both figures, the three components should add up to $100 \%$.

For fixed departures, as shown in Fig. 4, it is obvious that the percentage of schedule delay reduction increases monotonically as the level of unreliability increases, whereas the percentage of pure reliability cost reduction contribution (associated with the standard deviation) decreases with the unreliability level. The percentage of expected travel time cost reduction ranges from 25 to $62 \%$ across the levels of unreliability considered. This suggests that, if policy practitioners only take into account the expected travel time cost reduction, and ignore that from reliability improvement, the reduction in aggregate generalized user costs may be underestimated substantially, from 38 to $75 \%$.

For anticipating departures, as shown in Fig. 5, the variation of the percentage of schedule delay cost reduction is similar to the variation of the value of implied schedule delay shown in Table 2. The same explanation also applies here: when train services are extremely unreliable, travelers are more inclined to exhibit anticipating departures and, consequently, the expected schedule delay cost would be smaller than it would be with fixed departures. In this case, the percentage of expected travel time cost reduction ranges from 25 to $67 \%$ across various levels of unreliability. In other words, ignoring the reliability cost reduction will seriously underestimate the total reduction of aggregate generalized user cost, from 33 to $75 \%$. When we compare the percentage of expected travel time cost reduction between fixed and anticipating departures, the difference turns out to be noticeable when the unreliability level is large. This stems from the impact of anticipating departure behavior on the expected schedule delay costs when services are very unreliable. 
Fig. 4 Graphical illustrations for shares of the three components of generalized user cost reduction from a complete elimination of unreliability: fixed departures (a) Share of expected travel time cost reduction in aggregate generalized user cost reduction

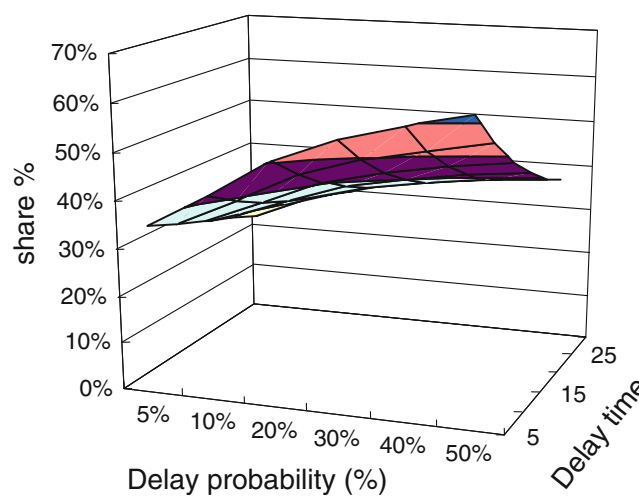

(b) Share of schedule delay cost reduction in aggregate generalized user cost reduction

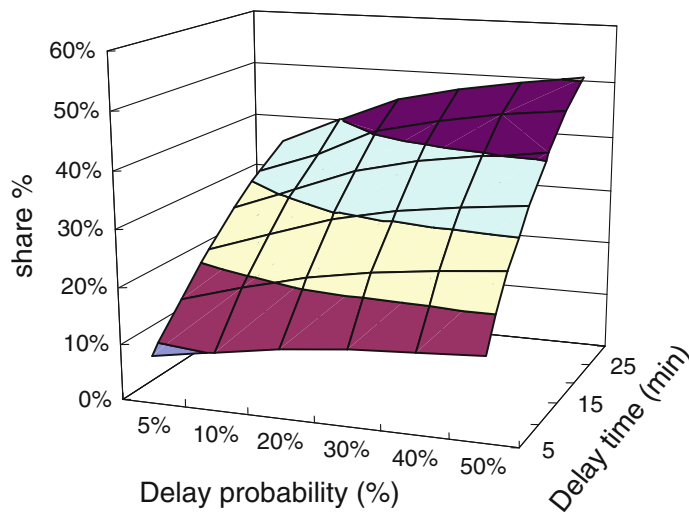

(c) Share of pure reliability cost reduction in aggregate generalized user cost reduction

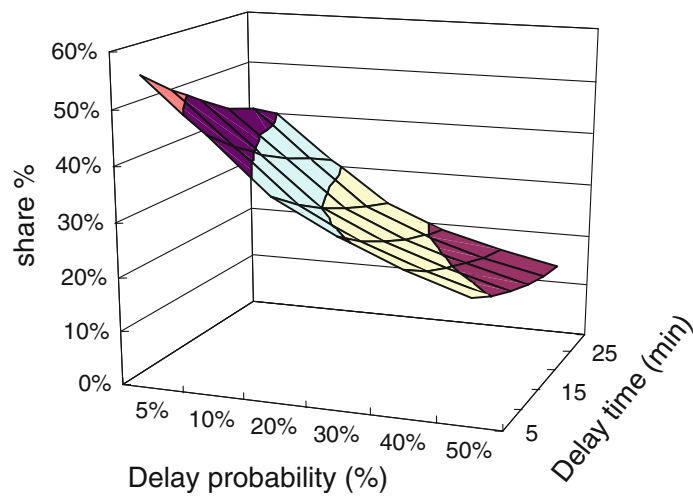

On the other hand, this also implies that, when the degree of unreliability is moderate, failing to take travelers' anticipating departures into account will not lead to much difference in the result of the generalized user cost reduction calculation. But ignoring 
Fig. 5 Graphical illustrations for shares of the three components of generalized user cost reduction from a complete elimination of unreliability: anticipating departures (a) Share of expected travel time cost reduction in aggregate generalized user cost reduction

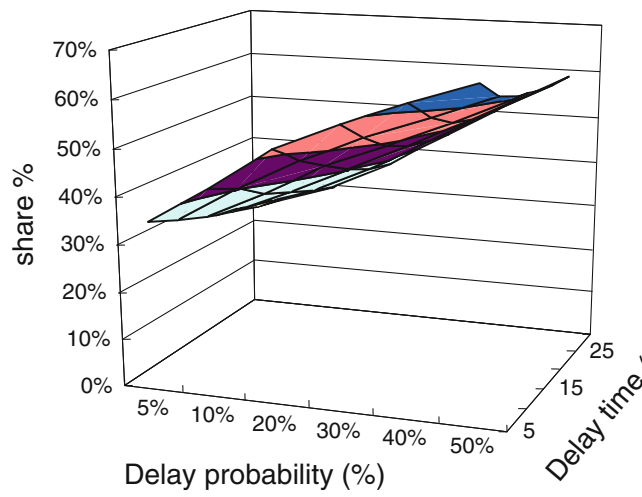

(b) Share of schedule delay cost reduction in aggregate generalized user cost reduction

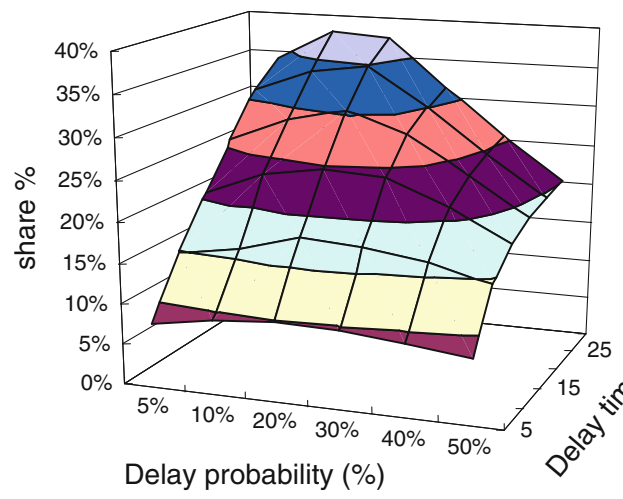

(c) Share of pure reliability cost reduction in aggregate generalized user cost reduction

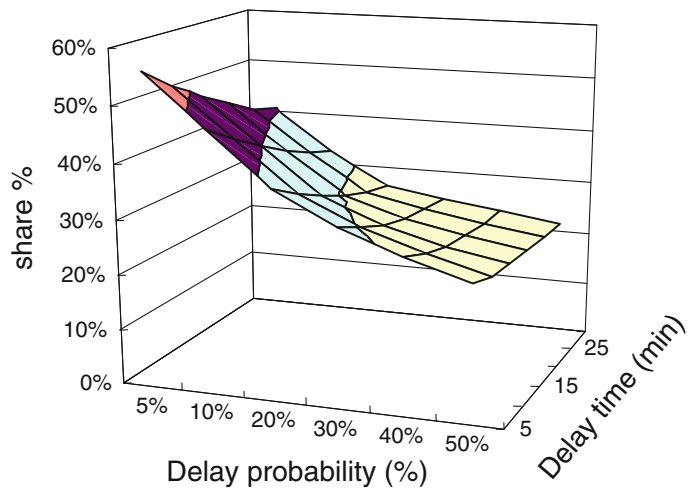

scheduled delay costs and costs of pure unreliability remains, of course, a potentially large source of underestimation of the generalized user cost savings.

Finally, our result in this section is comparable to what was found in Ettema and Timmermans (2006), who concluded that scheduling costs account for $20-40 \%$ of the 
generalized costs in the context of road transport. As shown in Tables 4 and 5, given a wide range of unreliability levels, the schedule delay costs contribute 8-50 and 8-38\% to the total cost reductions in the case of fixed and anticipating departures, respectively. It seems that our finding here is not far from the result of the analysis in Ettema and Timmermans (2006), even though these two studies concern rather different contexts (train versus car transport), and use different schedule delay parameters settings.

\section{Concluding remarks}

We established a simple model to describe and predict railway passengers' anticipating departure time behavior given their PATs, when choosing between scheduled train connection arrival times. Furthermore, we derived the expected schedule delay costs with and without considering this anticipating departure behavior for some specific (two masspoints) travel time distributions. The value of implied schedule delay can be related to the standard deviation of travel time distribution. The numerical examples in "Numerical illustrations" section provide some insight into how the anticipating departures and the values of implied schedule delay vary with the levels of unreliability.

In reality, public transport operators may be more interested in some travel time distributions than in others; our analysis can of course be applied to any multi-mass-points distribution so that a value can be determined for any specific application. The result can also be generalized by testing many possible (and reasonable) levels of unreliability, headways, and also different parameters of $\beta$ and $\gamma$, so that a good approximation of an average value of implied schedule delay can be obtained statistically.

The analysis in the final section focused on practical relevance. By exploring the relative sizes of cost reductions of three relevant components (expected travel time, schedule delays, and pure reliability), it was possible to identify the amount of bias incurred with a naïve CBA that only considers expected travel time costs. For our parameterization, the underestimation ranges from 33 to $75 \%$ of total generalized user cost savings across various unreliability levels when travelers' anticipating departure behavior is taken into account.

Note that our model was developed with in mind the case of intercity rail service, in which the headway is relatively long, and travelers' generalized time-related costs are sensitive to their departure time/connection choice. In the case of very frequent transit services, as provided within an urban area, travelers may not mind the connection choice as much as in the case of intercity rail travel, whilst they may simply add a buffer in the journey time to cope with the unforeseen delay. Therefore, the circumstance may be more similar to the timing of private car trips (where drivers can choose their departure time freely), or may be somewhere in between the situation of private car trips and the situation illustrated in our present model. For future research, it may be interesting to see the impact of unreliability on travelers' decisions under different travel circumstances.

There are some limitations to our analysis that we believe are also important to address in future work. The first is that our analysis is restricted to journeys with a single link, whereas multiple links are usually relevant in reality. Especially if journeys consist of several major links which are all unreliable, the situation may be much more complicated. Though some network model may be useful to calculate the compounded reliability, 
travelers' behavior may not be so easy to model, especially when some other disutilities such as waiting would also be involved. We believe this issue will remain challenging for future studies.

Secondly, in the present study we assume that the value of time, value of reliability, and value of schedule delay are homogeneous for all circumstances, whilst in reality these time-related values may vary quite a lot with, e.g., time of day, trip purpose, and travel circumstance. In the example of different time values by time of day, travelers may be penalized more by departing earlier than necessary due to the aversion of waking up early. Thus, accounting for heterogeneous time values by different circumstances may well be another interesting task for future work.

Acknowledgments This study was carried out as part of the Transumo project on Reliable Transport Chains. We are grateful for the research support received from ProRail and NS in the Netherlands. We would also like to thank two anonymous referees for very helpful comments on an earlier draft. The usual disclaimer applies.

Open Access This article is distributed under the terms of the Creative Commons Attribution Noncommercial License which permits any noncommercial use, distribution, and reproduction in any medium, provided the original author(s) and source are credited.

\section{Appendix 1}

Derivation for the value of implied schedule delay when the departure time is assumed to be fixed for both reliable and unreliable services

As discussed in "Unreliable train service and travelers' anticipating departure" section, a traveler will choose only connections $\mathrm{B}$ or $\mathrm{C}$ in the case of fully reliable services, and the switching level of $P A T^{*}=\frac{\gamma \cdot H}{\beta+\gamma}$ (given that $\theta=0$ ). It means that connection $\mathrm{B}$ is taken if a traveler's PAT falls in the interval $\left[0, \frac{\gamma \cdot H}{\beta+\gamma}\right]$, and connection $\mathrm{C}$ is taken otherwise. For the sake of simplicity, we will assume that $\theta=0$ in the derivation below.

The expected scheduling cost for reliable service is (as shown Eq. 7):

$$
E\left[D U_{s}\right]^{R}=\frac{1}{H}\left[\int_{0}^{\frac{\gamma \cdot H}{\beta+\gamma}} \beta \cdot P A T \cdot d P A T+\int_{\frac{\gamma \cdot H}{\beta+\gamma}}^{H} \gamma \cdot(H-P A T) \cdot d P A T\right]=\frac{\beta \cdot \gamma}{2(\beta+\gamma)} H .
$$

Suppose the traveler's departure time is fixed, regardless of the level of service reliability. In such a case, the switching point of PAT* is the same for both reliable and unreliable services. The expected scheduling costs for unreliable service can be derived separately under two situations:

(a) When $D T<P A T^{*}$, the expected scheduling cost of unreliable service is 


$$
E\left[D U_{s}\right]^{U}=\frac{1}{H}\left[\begin{array}{c}
\int_{0}^{D T}[\beta \cdot(1-p) \cdot P A T+\gamma \cdot p \cdot(D T-P A T)] \cdot d P A T \\
+\int_{D T}^{P A T *}[\beta \cdot(1-p) \cdot P A T+\beta \cdot p \cdot(P A T-D T)] \cdot d P A T \\
+\int_{P A T *}^{H} \gamma \cdot(H-P A T+p \cdot D T) \cdot d P A T
\end{array}\right] .
$$

Thus, the difference of scheduling cost between unreliable and reliable train services is:

$$
\Delta E\left[D U_{s}\right]=E\left[D U_{s}\right]^{U}-E\left[D U_{s}\right]^{R}=\frac{(\beta+\gamma)}{2 H} \cdot p \cdot D T^{2} .
$$

(b) When $D T \geq P A T^{*}$, the expected scheduling costs of unreliable service is:

$$
E\left[D U_{s}\right]^{U}=\frac{1}{H}\left[\begin{array}{l}
\int_{0}^{P A T *}[\beta \cdot(1-p) \cdot P A T+\gamma \cdot p \cdot(D T-P A T)] \cdot d P A T \\
+\int_{P A T *}^{H} \gamma \cdot(H-P A T+p \cdot D T) \cdot d P A T
\end{array}\right] .
$$

Thus, the difference in scheduling costs between unreliable and reliable train services is:

$$
\Delta E\left[D U_{s}\right]=E\left[D U_{s}\right]^{U}-E\left[D U_{s}\right]^{R}=\gamma \cdot p \cdot D T-\frac{\gamma^{2}}{2(\beta+\gamma)} \cdot p \cdot H
$$

Derivation of the value of implied schedule delay when the anticipating departure is taken into account for the unreliable service

When the traveler's behavior change, i.e. anticipating departure, is taken into account, the switching level of PAT for unreliable services is different from the case for reliable services. Furthermore, connection A becomes one of the available choices among the alternative connections in the unreliable services, such that there are two switching levels of PAT in this case. Here, we define $P A T^{\prime}$ as the switching point between A and B, and $P A T^{\prime \prime}$ as the switching point between $\mathrm{B}$ and $\mathrm{C}$.

The switching level of PAT between connections A and B is:

$$
P A T^{\prime \prime}=D T-\frac{\beta \cdot H}{(\beta+\gamma) \cdot p} .
$$

The switching level of PAT between connections B and C is:

$$
P A T^{\prime}=\frac{\gamma \cdot H}{\beta+\gamma}+p \cdot D T .
$$

For this unreliable train service, there is a critical level of delay time, denoted as $D T^{\prime}$, such that, when $D T<D T^{*}$, connection A is always worse-off than connection $\mathrm{B}$, whilst when 
$D T \geqq D T^{*}$, connection $\mathrm{C}$ is always worse-off than connection $\mathrm{B}$. This critical delay time can be obtained as:

$$
D T^{*}=\frac{\beta \cdot H}{(\beta+\gamma) \cdot p} .
$$

Thus, when $D T<D T^{*}$, the traveler will only choose between connections B and $\mathrm{C}$, and, when $D T \geqq D T^{*}$, the traveler will therefore only choose between connections A and B. The expected scheduling costs for this unreliable service can therefore be computed separately for these two situations:

(a) When $D T<D T^{*}$, connections B and C will be chosen, the expected scheduling cost for unreliable service is:

$$
E\left[D U_{s}\right]^{U}=\frac{1}{H}\left[\begin{array}{l}
\int_{0}^{D T}[\beta \cdot(1-p) \cdot P A T-\gamma \cdot p \cdot(D T-P A T)] \cdot d P A T \\
+\int_{D T}^{P A T^{\prime}}[\beta \cdot(1-p) \cdot P A T+\beta \cdot p \cdot(P A T-D T)] \cdot d P A T \\
+\int_{P A T^{\prime}}^{H} \gamma \cdot(H-P A T+p \cdot D T) \cdot d P A T
\end{array}\right] .
$$

The difference in scheduling cost between unreliable and reliable train services is:

$$
\Delta E\left[D U_{s}\right]=E\left[D U_{s}\right]^{U}-E\left[D U_{s}\right]^{R}=\frac{(\beta+\gamma)}{2 H} \cdot p \cdot(1-p) \cdot D T^{2} .
$$

(b) When $D T \geqq D T^{*}$, connections A and B will be chosen, and the expected scheduling cost for unreliable service is:

$$
E\left[D U_{s}\right]^{U}=\frac{1}{H}\left[\begin{array}{c}
\int_{0}^{P A T^{\prime \prime}} \beta \cdot(P A T+H-p \cdot D T) \cdot d P A T \\
+\int_{P A T^{\prime \prime}}^{D T}[\beta \cdot(1-p) \cdot P A T+\gamma \cdot p \cdot(D T-P A T)] \cdot d P A T \\
+\int_{D T}^{H}[\beta \cdot(1-p) \cdot P A T+\beta \cdot p \cdot(P A T-D T)] \cdot d P A T
\end{array}\right] .
$$

The difference in scheduling costs between unreliable and reliable train services is:

$$
\Delta E\left[D U_{s}\right]=E\left[D U_{s}\right]^{U}-E\left[D U_{s}\right]^{R}=\beta \cdot\left(\frac{1}{2} H+(1-p) \cdot D T-\frac{\beta \cdot H}{2(\beta+\gamma) \cdot p}\right)-\frac{\beta \cdot \gamma \cdot H}{2(\beta+\gamma)} .
$$




\section{Appendix 2}

See Appendix Tables 4, 5.

Table 4 Shares of the three components of generalized user cost reduction from a complete elimination of unreliability: fixed departures

\begin{tabular}{|c|c|c|c|c|c|c|}
\hline \multirow[t]{2}{*}{ Delay time (min) } & \multicolumn{6}{|c|}{ Delay probability } \\
\hline & $5 \%$ & $10 \%$ & $20 \%$ & $30 \%$ & $40 \%$ & $50 \%$ \\
\hline \multicolumn{7}{|c|}{ Shares of expected travel time cost reduction } \\
\hline 5 & 35.42 & 42.93 & 50.90 & 55.77 & 59.42 & 62.46 \\
\hline 10 & 32.68 & 38.99 & 45.43 & 49.28 & 52.10 & 54.42 \\
\hline 15 & 30.34 & 35.70 & 41.02 & 44.14 & 46.39 & 48.22 \\
\hline 20 & 28.32 & 32.92 & 37.40 & 39.97 & 41.81 & 43.26 \\
\hline 25 & 25.54 & 30.93 & 34.36 & 36.52 & 38.05 & 39.27 \\
\hline 30 & 25.15 & 28.72 & 32.07 & 33.94 & 35.26 & 36.30 \\
\hline \multicolumn{7}{|c|}{ Shares of schedule delay cost reduction } \\
\hline 5 & 8.42 & 10.21 & 12.07 & 13.24 & 14.10 & 22.72 \\
\hline 10 & 15.48 & 18.46 & 21.52 & 23.34 & 24.68 & 25.78 \\
\hline 15 & 21.54 & 25.34 & 29.13 & 31.33 & 32.94 & 34.24 \\
\hline 20 & 26.79 & 31.15 & 35.39 & 37.82 & 39.56 & 41.00 \\
\hline 25 & 31.38 & 35.31 & 40.64 & 43.19 & 45.00 & 46.44 \\
\hline 30 & 34.97 & 39.94 & 44.60 & 47.20 & 49.03 & 50.49 \\
\hline \multicolumn{7}{|c|}{ Shares of pure reliability cost reduction } \\
\hline 5 & 56.16 & 46.86 & 37.03 & 30.99 & 26.48 & 14.82 \\
\hline 10 & 51.84 & 42.55 & 33.05 & 27.38 & 23.22 & 19.80 \\
\hline 15 & 48.12 & 38.96 & 29.85 & 24.53 & 20.67 & 17.54 \\
\hline 20 & 44.89 & 35.93 & 27.21 & 22.21 & 18.63 & 15.74 \\
\hline 25 & 43.08 & 33.76 & 25.00 & 20.29 & 16.95 & 14.29 \\
\hline 30 & 39.88 & 31.34 & 23.33 & 18.86 & 15.71 & 13.21 \\
\hline
\end{tabular}

Table 5 Shares of the three components of generalized user cost reduction from a complete elimination of unreliability: anticipating departures

\begin{tabular}{|c|c|c|c|c|c|c|}
\hline \multirow[t]{2}{*}{ Delay time $(\mathrm{min})$} & \multicolumn{6}{|c|}{ Delay probability } \\
\hline & $5 \%$ & $10 \%$ & $20 \%$ & $30 \%$ & $40 \%$ & $50 \%$ \\
\hline \multicolumn{7}{|c|}{ Shares of expected travel time cost reduction } \\
\hline 5 & 35.58 & 43.40 & 52.18 & 58.10 & 63.00 & 67.48 \\
\hline 10 & 32.95 & 40.64 & 47.49 & 53.00 & 57.83 & 62.71 \\
\hline 15 & 30.68 & 36.64 & 43.57 & 48.78 & 54.53 & 60.57 \\
\hline 20 & 28.70 & 33.99 & 40.26 & 46.30 & 53.00 & 59.56 \\
\hline 25 & 26.68 & 31.70 & 37.73 & 44.94 & 52.13 & 58.97 \\
\hline 30 & 25.50 & 29.72 & 36.20 & 44.07 & 51.56 & 58.58 \\
\hline
\end{tabular}


Table 5 continued

\begin{tabular}{|c|c|c|c|c|c|c|}
\hline \multirow[t]{2}{*}{ Delay time (min) } & \multicolumn{6}{|c|}{ Delay probability } \\
\hline & $5 \%$ & $10 \%$ & $20 \%$ & $30 \%$ & $40 \%$ & $50 \%$ \\
\hline \multicolumn{7}{|c|}{ Shares of schedule delay cost reduction } \\
\hline 5 & 7.99 & 9.23 & 9.86 & 9.61 & 8.93 & 7.97 \\
\hline 10 & 14.80 & 16.00 & 17.96 & 17.54 & 16.40 & 14.47 \\
\hline 15 & 20.67 & 23.38 & 24.72 & 24.12 & 21.17 & 17.39 \\
\hline 20 & 25.78 & 28.92 & 30.45 & 27.97 & 23.38 & 18.77 \\
\hline 25 & 31.03 & 33.71 & 34.82 & 30.09 & 24.64 & 19.58 \\
\hline 30 & 34.05 & 37.85 & 37.47 & 31.44 & 25.47 & 20.11 \\
\hline \multicolumn{7}{|c|}{ Shares of pure reliability cost reduction } \\
\hline 5 & 56.43 & 47.37 & 37.96 & 32.29 & 28.07 & 24.55 \\
\hline 10 & 52.25 & 43.36 & 34.55 & 29.46 & 25.77 & 22.82 \\
\hline 15 & 48.65 & 39.98 & 31.71 & 27.10 & 24.30 & 22.04 \\
\hline 20 & 45.52 & 37.09 & 29.29 & 25.73 & 23.62 & 21.67 \\
\hline 25 & 42.29 & 34.59 & 27.45 & 24.97 & 23.23 & 21.45 \\
\hline 30 & 40.45 & 32.43 & 26.33 & 24.49 & 22.97 & 21.31 \\
\hline
\end{tabular}

\section{References}

Bates, J., Polak, J., Jones, P., Cook, A.: The valuation of reliability for personal travel. Transp. Res. E 37(2-3), 191-229 (2001)

Black, I.G., Towriss, J.G.: Demand Effects of Travel Time Reliability. Center for Logistics and Transportation, Cranfield Institute of Technology, Cranfield (1993)

de Palma, A., Lindsey, R.: Optimal timetables for public transportation. Transp. Res. B 35(8), 789-813 (2001)

Ettema, D., Timmermans, H.: Costs of travel time uncertainty and benefits of travel time information: conceptual model and numerical examples. Transp. Res. C 14(5), 335-350 (2006)

Fosgerau, M.: The marginal social cost of headway for a scheduled service. Transp. Res. B 43(8-9), 813-820 (2009)

Fosgerau, M., Karlström, A.: The value of reliability. Transp. Res. B 44(1), 38-49 (2010)

Gaver Jr., D.P.: Headstart strategies for combating congestion. Transp. Sci. 2(2), 172-181 (1968)

Gómez-Ibáñez, J.A., Tye, W., Winston, C. (eds.): Essays in Transportation Economics and Policy: Handbook in Honor of John R. Meyer. Brookings Institution, Washington, DC (1999)

Knight, T.E.: An approach to the evaluation of changes in travel time unreliability: a "safety margin". Hypothesis Transp. 3(4), 393-408 (1974)

Noland, R.B., Small, K.A.: Travel time uncertainty, departure time and the cost of the morning commute. Transp. Res. Rec. 1493, 150-158 (1995)

Polak, J.: Travel time variability and departure time choice: a utility theoretic approach. Discussion paper No. 15, Transport Studies Group, Polytechnic of London (1987)

Small, K.A.: The scheduling of consumer activities: work trips. Am. Econ. Rev. 72(3), 467-479 (1982)

Tseng, Y.-Y.: Valuation of travel time reliability in passenger transport. Dissertation, Tinbergen Institute Research Series 439, Vrije Universiteit Amsterdam (2008)

Vickrey, W.S.: Congestion theory and transport investment. Am. Econ. Rev. 59, 251-260 (1969)

Wardman, M.: Public transport values of time. Transp. Pol. 11(4), 363-377 (2004) 


\section{Author Biographies}

Yin-Yen Tseng holds a post-doc position at the department of Spatial Economics, VU University Amsterdam. She obtained her PhD degree with the thesis entitled "Valuation of travel time reliability in passenger transport" at the VU University Amsterdam in 2008. Her research themes include discrete choice modeling, travel behavior, and valuation.

Piet Rietveld is Professor in Spatial economics at VU University, Amsterdam. He studied econometrics and is a fellow of the Tinbergen Institute. His research interests are in the fields of travel behavior, transport policy, transport and adaptation to climate change, transport and spatial development.

Erik T. Verhoef is Professor of Spatial Economics at the VU University Amsterdam and research fellow of the Tinbergen Institute. His research focuses on transport themes such as second-best policies in transport network models, network modeling, and valuation. He is on the editorial boards of various journals including Transportation Research B, Journal of Urban Economics, and Journal of Transport Economics and Policy. 